This item was submitted to Loughborough's Research Repository by the author.

Items in Figshare are protected by copyright, with all rights reserved, unless otherwise indicated.

\title{
Cognitive enhancement of numerical and arithmetic capabilities: a mini- review of available transcranial electric stimulation studies
}

PLEASE CITE THE PUBLISHED VERSION

https://doi.org/10.1007/s41465-016-0006-z

\section{PUBLISHER}

Springer Science and Business Media LLC

\section{VERSION}

AM (Accepted Manuscript)

\section{PUBLISHER STATEMENT}

This is a post-peer-review, pre-copyedit version of an article published in Journal of Cognitive Enhancement. The final authenticated version is available online at: https://doi.org/10.1007/s41465-016-0006-z

\section{LICENCE}

CC BY-NC-ND 4.0

\section{REPOSITORY RECORD}

Schroeder, PA, T Dresler, Julia Bahnmueller, C Artemenko, R Cohen Kadosh, and H-C Nuerk. 2017. "Cognitive Enhancement of Numerical and Arithmetic Capabilities: A Mini-review of Available Transcranial Electric Stimulation Studies”. Loughborough University. https://hdl.handle.net/2134/13109573.v1. 
Cognitive enhancement of numerical and arithmetic capabilities:

\section{A short review of available transcranial electric stimulation studies}

Schroeder, P. A. ${ }^{a, b^{*}}$, Dresler, T. ${ }^{a, d}$, Bahnmueller, J. ${ }^{b, c}$, Artemenko, C. ${ }^{b, d}$, Cohen

Kadosh, R. ${ }^{e}$, Nuerk, H.-C. ${ }^{b, c, d}$

\section{Abbreviated title: \\ Cognitive enhancement of arithmetic capabilities}

Word Count: 3211 words (max: 3300 )

Number of Tables: 1

Number of Figures: 0

\section{Address:}

a Department of Psychiatry and Psychotherapy, University of Tübingen, Calwerstr. 14, 72076 Tübingen, Germany

b Department of Psychology, University of Tübingen, Schleichstr. 4, 72076 Tübingen, Germany

c Leibniz-Institut für Wissensmedien, Schleichstr. 4, 72076, Tübingen, Germany

d LEAD Graduate School \& Research Network, University of Tübingen, Gartenstr. 29, 72074 Tübingen, Germany

e Department of Experimental Psychology, University of Oxford, Oxford 0X1 3UD,

United Kingdom

\section{Corresponding author}

Dipl.-Psych. Philipp A. Schroeder

Department of Psychiatry \& Psychotherapy

Neurophysiology \& Interventional Neuropsychiatry

University of Tübingen

Calwerstraße 14

D-72076 Tübingen, Germany

Tel: +49 7071-29-8 0815

Fax: +49 7071-29-59 04

E-Mail: philipp.schroeder@uni-tuebingen.de 


\begin{abstract}
Arithmetic capabilities are complex cognitive skills essential for handling requirements of the modern world. At the same time, educational institutions are challenged with math-related problems, e.g., developmental dyscalculia, math anxiety, and also with less severe difficulties of arithmetic understanding. Thus, noninvasive techniques for cognitive enhancement have attracted researchers' and practitioners' interest in the fields of education, psychology, and neuroscience. Particularly, studies employing transcranial electric stimulation (tES) in arithmetic learning, problem solving, and performance in numerical tasks and operations have shaped an optimistic perspective of cognitive enhancement in these domains, building on the fronto-parietal correlates of healthy and deficient arithmetic performance and learning. However, the heterogeneity of stimulation approaches in numerical cognition research - with different electrode montages, stimulation protocols, tasks, outcomes, and combinations thereof - may also showcase a variety of parameters relevant more generally to the cognitive domain. Here we present a short overview of the different tES approaches to enhance arithmetic capabilities within the general framework of cognitive enhancement. We conclude that performance and training gains can be obtained from different strategical tES configurations, but more standardization, better translation between neurodevelopmental perspectives and tES principles, and controlled studies in critical populations are needed.
\end{abstract}

Keywords: transcranial direct current stimulation, transcranial random noise stimulation, cognitive enhancement, numerical cognition, educational neuroscience 
Basic arithmetic abilities are critical for numerous activities and societal functioning. Yet, a substantial amount of the general population (e.g., up to $22 \%$ in the UK) shows mathematical deficits which are often specified in developmental trajectories, cognitive disabilities, or comorbidities (Kaufmann et al. 2013) and which can result in occupational and economic disadvantages (Bynner and Parsons 1997). Both domain-general and domain-specific functions supplement the successful operation on numerical quantities in everyday life. These cognitive functions include magnitude representation, retrieval of, and operation on arithmetic facts on the domain-specific side, and working memory, executive functions, and attention on the domain-general side. The variety of involved domain-general and domain-specific cognitive functions thus also increases the dimensionalities of possible mathematics training strategies (Looi and Cohen Kadosh 2016).

Arithmetic processing is mainly subserved by the fronto-parietal network of the brain (Klein et al. 2014; Matejko and Ansari 2015; Nieder 2016): Different parietal circuits are particularly relevant for magnitude representations (Dehaene et al. 2003) and arithmetic operations often produce additional prefrontal activations (Arsalidou and Taylor 2011). By modulating activity in these brain areas, arithmetic performance could be changed. Accordingly, investigations with transcranial brain stimulation can causally bolster the correlational evidence from neuroimaging, because brain activity becomes an independent manipulated variable rather than a dependent, measurable variable. Going beyond causal reasoning, neuromodulation has also been proposed as effective strategy to improve arithmetic capabilities over and above behavioral cognitive training.

In particular, transcranial electric stimulation (tES) has been administered repeatedly in recent studies using various arithmetic tasks and trainings. However, the complex neurocognitive functions required in different arithmetic tasks have sparked different approaches within tES studies. Moreover, the neuromodulatory technique itself also allows for different implementations with critical consequences for assumed neurophysiological and behavioral effects. Thus, heterogeneity (anatomically, parametrically, and content-wise) is the norm and it is hard to get a conclusive overview even on this small field. Therefore, we present a short review of studies employing transcranial electric stimulation (tES) on numerical processing and learning disentangling above factors. Our particular focus is to discuss basic methodological and translational aspects of tES approaches in the general 
framework of cognitive enhancement that may contribute to the current heterogeneity.

\section{Stimulation for enhancing arithmetic capabilities}

The current review focuses on tES methods, but numerical cognition research is also informed by extensive previous work with transcranial magnetic stimulation (TMS) essentially testing the causal structure-function relations underlying arithmetic skills with high focality (Salillas and Semenza 2015). Some advantages of administering weak currents through scalp electrodes with tES over active interference with electromagnetic TMS pulses are the possibility of modulating brain activity online (that is: concurrent to a behavioral task or training) without auditory and sensory distraction, the establishment of sham and active control stimulation with indistinguishable sensory artifacts (see also Duecker and Sack 2015), portability, economic efficiency, and easy implementation in outpatient interventions (Priori et al. 2009).

Within tES applications for the enhancement of numerical cognition, the most prominently investigated methods are transcranial direct current stimulation (tDCS) and transcranial random noise stimulation (tRNS). For both techniques, two (or more) surface electrodes are fixed over brain regions and a weak current is applied (mostly 1-2 mA). Using direct currents in tDCS, cathode and anode electrodes differ in their neurophysiological effect. It is assumed that in the human brain underneath the anode, depolarization mostly produces excitatory shifts of resting membrane potentials whereas underneath the cathode, hyperpolarization mostly modulates neuronal activity in inhibitory ways (Nitsche and Paulus 2000; but see Jacobson et al. 2012 for review of polarity effects). In contrast to TMS, these subtle neuromodulatory shifts are not capable to induce action potentials on their own, but tES can emphasize or attenuate inherent neural activity and thus also produce cross-cortical network responses. Any modulation of behavioral effects thus rests on the current network activity (state-dependency) and on neural activations as induced by a task (Silvanto and Pascual-Leone 2008; Bikson and Rahman 2013).

Often, only the effect under one 'target' electrode is desirable. However, 'return' electrode placement (typically of the cathode) is highly relevant, because its placement also affects current flow and current fields underneath the target electrode, e.g., due to target-return distance and direction, shunting over the scalp, or 
network dynamics (Bikson et al. 2010; Moliadze et al. 2010). In addition, regions between the electrodes are flooded by tangential current, which can modulate additional synaptic efficiency of some neuronal populations (Rahman et al. 2013). This is a particular problem in numerical cognition, because - as outlined above domain-general functions also contribute to numerical functioning. It is not sufficient to avoid number-related brain areas for the placement of the return electrode, because a domain-general function underlying the overall current flow may also exert its influence on numerical processing. Furthermore, an opposite polarization in another brain region may be of theoretical interest (e.g., in oppositional placements). However, in most cases of unilateral placement with one theoretically motivated target electrode, the return and intermediate area activity is neglected and accordingly, large return pads are used that produce less dense current fields or return electrodes are placed on extracephalic locations.

In contrast, alternating currents are used in tRNS with (high) frequencies randomly picked from a normally distributed range of predefined oscillations. What is known about tRNS mechanisms is that its intensity- and frequency-dependent administration can enhance cortical excitability underneath both surface electrodes (Terney et al. 2008) and tRNS can facilitate subthreshold detection processes according to stochastic resonance principles (Antal and Herrmann 2016; van der Groen and Wenderoth 2016). Note that tRNS working mechanisms differ from the fixed application of a single oscillation in transcranial alternating current stimulation (tACS), which is thought to act by entrainment and phase-locking (Ozen et al. 2010; Battleday et al. 2014). The excitatory effect of tRNS tended to be larger, yet shorter, compared to anodal tDCS, in motor cortex evaluations (Moliadze et al. 2014). In cognitive tasks, mixed results were obtained with effective modulations in numerical tasks and trainings (Cappelletti et al. 2013; Snowball et al. 2013; Pasqualotto 2016; Popescu et al. 2016), steeper learning rates - as opposed to anodal tDCS - in visual discrimination (Fertonani et al. 2011), but no performance modulations in working memory tasks (Mulquiney et al. 2011). Little direct behavioral outcome comparisons between the two techniques exist to date. Yet, their different characteristics could imply better targeted implementations: For instance, the aftereffects of tRNS appear NMDA receptor independent, in contrast to anodal tDCS (Chaieb et al. 2015). Thus, different neuroplastic (long-term) consequences of tRNS and tDCS could further augment and specify future training effects in different conditions. 
------- PLEASE INSERT TABLE 1 ABOUT HERE

\section{Short overview of reviewed studies}

Acknowledging the involvement and relevance of different domain-specific and domain-general functions, associated brain areas, and networks in numeracy and arithmetic processes exceeds the scope of this mini-review, but good and elaborated descriptions are available elsewhere (Arsalidou and Taylor 2011; Looi et al. 2016b). So far, various combinations of tasks, study designs, and electrode configurations have been investigated using tES methods in single studies. Table 1 presents a systematic overview of the reviewed studies ${ }^{1}$. Here, we discuss the heterogeneity of approaches towards interactions between stimulation parameters and tasks assessing numerosity and arithmetic performance and learning.

\section{Numerical performance modulations}

The available study results corroborate the importance of bilateral parietal brain regions for numerical processing, but active control stimulations and tasks also indicate distinct lateralization and state-dependency.

In number magnitude comparison tasks, unilateral left-side parietal anodal tDCS generally increased accuracy with two-digit number symbols (Hauser et al. 2013). Conversely, a bipolar oppositional placement with right anodal, left cathodal parietal stimulation impaired single-digit comparison latencies ( $\mathrm{Li}$ et al. 2015) for digits close to the referent. Thus, the bipolar stimulation modulated the increasing difficulty to distinguish digits closer to the comparison referent (numerical distance effect (NDE); Moyer and Landauer 1967). Considering the state-dependency principle of tES effects, this modulation of NDE could be either domain-specific or it could be driven by the higher level of task difficulty and neural activation in critical trials. In other words, higher demands for discriminating close targets and for processing multi-digits could encompass more task-induced activity ready for neuromodulation. NDE was

\footnotetext{
${ }^{1}$ A PubMed search (tDCS/tRNS AND arithmetic/numerical cognition) identified 34 papers that
} were manually screened for original results and new cross-referenced studies. 
unaffected by several tDCS configurations of Hauser et al. (2013) in multi-digit comparisons, but the distractor-distance effect for selecting correct two-digit addition results was monotonically modulated by a bilateral-bicephalic parietal stimulation (Klein et al. 2013). Both single-digit accuracy rates and latencies as well as NDE were unaffected by prefrontal cathodal tDCS, but spatial-numerical associations were eliminated (Schroeder et al. 2016).

\section{Arithmetic performance modulations}

Performing even on simple operations requires domain-general working memory and executive functions involvement to maintain operation components, yet to different extents depending on the exact task. Most studies employed supraorbital or prefrontal return cathodes without necessarily highlighting potential effects on such domain-general functions. Interestingly, for novel and complex subtractions, leftparietal tDCS prevented characteristic prefrontal deactivations below the rightprefrontal cathode as captured with simultaneous fMRI (Hauser et al. 2016), whereas a behavioral effect for subtraction problems was only found in a preceding study (Hauser et al. 2013). Similarly, left-parietal performance enhancements in a complex statistical procedure were observed with temporal (Houser et al. 2015), but not with prefrontal cathode placement (Houser et al. 2014). Also considering neurodevelopmental studies, the accumulation of arithmetic proficiency may be accompanied by a shift from broad prefrontal to precise parietal activations (Zamarian et al. 2009). In this line, both frontal and parietal excitatory tRNS generated greater speed improvements over the course of an experiment in subtraction verifications, but not in word classifications (Pasqualotto 2016). Furthermore, tDCS to one area can lead to altered activations over wide-spread networks in the brain and to effects on cognitive functions related to areas distant from the stimulated area. For example, two studies report improvements in paced auditory serial subtraction tasks from cathodal stimulation of the cerebellum, thought to disinhibit prefrontal activity (Pope and Miall 2012), and comparable results were obtained with prefrontal anodal tDCS (Pope et al. 2015). In clinical research, a variant of the serial addition WM task is often used in conjunction with tDCS to enhance domain-general functions and emotional processing in depressive patients (Vanderhasselt et al. 2015). Solving repeated additions with adaptive speed increases is frustrating and elicits negative affect, but anodal prefrontal tDCS can 
concurrently improve emotional responses and arithmetic performance (Plewnia et al. 2015). Highlighting individual differences, low- and high-math anxiety individuals responded differentially with decreased and increased arithmetic performance to prefrontal tDCS (Sarkar et al. 2014). These finding also highlight the importance of emotion for math-related cognitive processing, for which behavioral correlations have often been reported (Suárez-Pellicioni, Macarena, Núñez-Peña, María Isabel, \& Colomé 2016).

Also domain-specific effects in multi-digit processing could be modulated: Leftparietal anodal tDCS with $1.5 \mathrm{~mA}$ was beneficial for solving problems with larger operands (problem size effect; sums exceeding 10 and including carries), but decreased accuracy in small problems (Rütsche et al. 2015). Whereas the latter result is counterintuitive, system noise injection by anodal tDCS (see also Fertonani and Miniussi 2016) could skew arithmetic retrieval precisions and this conception was corroborated by concurrent EEG theta measurement results (Rütsche et al. 2015). Regarding place-value effects (e.g., carrying unit-digits), right-side anodal vs. cathodal parietal tDCS prolonged latency increases for carry operations in a two-digit addition task out of a series of unilateral placements (Artemenko et al. 2015).

\section{Numerical training modulations}

Whereas tRNS was used only selectively in studies on performance, the technique appears more appealing for numerical and arithmetic training. Highlighting the role of concurrent ('online') activities, Cappeletti et al. (2013) assembled comparisons between the combinations of training with parietal, motor, and sham tRNS over five days, as well as with a resting-state parietal stimulation. Number acuity in dot-array discrimination improved most from the parietal stimulation combined with training, but no transfer to arithmetic tasks was observed.

To investigate numerosity learning, a prominently investigated paradigm is the artificial symbols training where arbitrary, meaningless figures are assigned to numerical magnitudes. Repeated feedback-guided magnitude comparisons with the artificial symbols (training phase) produce automatic numerosity evaluations that interfere with physical size presentations and map onto space (test phase; Tzelgov et al. 2000). An oppositional parietal tDCS placement (right anodal, left cathodal) produced faster automaticity and more linear mapping of artificial symbols in a training over 6 days, with sustainability after 6 months (Cohen Kadosh et al. 2010). 
For the opposite placement, an impaired automaticity was detected, but here the bilateral parietal stimulation also produced steeper learning curves superior to prefrontal and sham stimulations (Iuculano and Cohen Kadosh 2013). Interestingly, when the numerical training paradigm was administered in two individuals with severe arithmetic problems, only the left-anodal right-cathodal configuration, which led to impairment in typical participants, led to behavioral improvements (luculano and Cohen Kadosh 2014). Although certainly larger samples are required to confirm this pattern, the application of tDCS-combined numerical trainings may provide effective rehabilitation and treatment prospects for numerical deficits.

\section{Arithmetic training modulations}

With artifical symbols depicting calculation algorithms for typical Arabic numbers, drill and calculation training combined with prefrontal tRNS improved learning rates and led to sustained calculation performance 6 months later (Snowball et al. 2013). In addition, hemodynamic recordings captured short-term and long-term physiological tRNS effects. More recently, an interesting combination of prefrontal tRNS on days 13 and parietal tRNS on days 4-5 was observed to improve performance in difficult math problems and accuracy in new and easy problems (Popescu et al. 2016). But also targeting the left parietal tDCS with $1.5 \mathrm{~mA}$ in a single-day training study on multiplication and subtraction facts, polarity-specific and operation-specific subtraction learning improvements were found and performance differences were sustained in $24 \mathrm{~h}$ follow-up (Grabner et al. 2015).

In combination with an adaptive body-tracking video-game on fractions, prefrontal bipolar-balanced tDCS (left anodal, right cathodal) resulted in improved and sustained task performance and transferred also to domain-general functions (Looi et al. 2016a).

These first training studies demonstrate the potential of tES for enhancing arithmetic training effects in healthy adult subjects. Evaluation numerical training studies with tDCS and tRNS already incorporate assessments of additional informative indices such as learning curves, specific and general transfer to non-trained stimuli and tasks, long-term effects, and neurophysiological profiles, although effects on domaingeneral functions are less well discriminated. In contrast, studies employing tESaugmented trainings in atypical populations are scarce and potentially restrained by additional physical and cognitive side effects (Krause and Cohen Kadosh 2013). 
Different neurophysiological profiles of individuals or groups (e.g., dyscalculia, stroke patients, children) at certain development stages most likely necessitate parametrical and training-related adjustments, e.g., to also consider specific numerical, arithmetic, and domain-general disadvantages such as working memory capacity. Moreover, most conclusions are based on single studies, researchers implement dissimilar tES configurations, and the interdependence of different brain areas and effects in certain tasks is not completely clear. In order to further integrate these partially heterogeneous findings, interpretations of different modulations by tES must also consider and build on the ongoing validation of its very basic principles.

\section{tES Configurations}

According to physical arrangements classification (Nasseri et al. 2015), successful modulations of numerical performance utilized unilateral monopolar placements with large return electrodes, bilateral bipolar-balanced and non-balanced, as well as the dual-channel bilateral double-monopolar arrangement by Klein et al. (2013). Most studies focus on parietal placements with $1 \mathrm{~mA}$, but also prefrontal placements appear at least partially successful with tRNS (Pasqualotto 2016) and produce significant modulations of spatial-numerical associations (Schroeder et al. 2016) and improvements in arithmetic decisions for high-math anxiety individuals (Sarkar et al. 2014).

All studies with tRNS used a bilateral bipolar-balanced placement with electrodes either targeting left and right prefrontal areas (e.g., F3 and F4) or parietal areas (e.g., P3 and P4). We wish to highlight once more that these placements are taken to increase excitability over both targeted areas and there is no need of an additional return electrode. Thus, in contrast to tDCS, but similar to placements with two pairs of tDCS electrodes (cf. Klein et al., 2013), the bipolar-balanced placement in tRNS is less likely to modulate hemispheric activity dominance and may prove useful for bilateral magnitude processing. Yet, its exact neurophysiological principles are different from (anodal) tDCS and currently not completely understood (Antal and Herrmann 2016).

An interesting concept is the modulation of distinct learning phases over different cortex regions as implemented by Popescu et al. (2016). Future research needs to validate this approach by closed-loop tDCS or by comparing different configuration changes directly (e.g., switching from prefrontal to parietal stimulation after 2, 3, or 4 
days). The potential of an augmentative effect and better targeting of stimulation by considering training phase is also confirmed by the finding that a specific tDCS polarity sequence can lead to more effective modulations with long-term sustainability (Dockery et al. 2009). Thus, basic research can also inform interventional administrations, although generalizations from healthy volunteers to different clinical populations (e.g., developmental dyscalculia or acalculia after a stroke) should consider all translational aspects (e.g., functional, structural, and behavioral differences) in corresponding models before selecting tES configuration parameters. Technical tES parameters have important connotations for behavioral effects. In the literature, prominently debated tES parameters are electrode configuration and sizes, current intensity and duration, and timing of stimulation (online vs. offline). These and other parameters do not work linearly (e.g., higher intensities can exceed optimal stimulation ranges; Batsikadze et al. 2013) and their combination outcomes might critically depend on task and individual characteristics. Eventually, this variety of possibilities will allow for broad-ranging applications and fine-grained targeting from underlying theoretical models. Currently, however, simplification or even standardization of certain parameters would facilitate effectivity estimations, and the ongoing development of electric field modelling tools can already be used for selecting stimulation targets (Truong et al. 2014). Regarding numerical processes, stimulations should be administered concurrent to a task and could then achieve neurostructural precision following task-selective recruitment (Clemens et al. 2013; Bikson and Rahman 2013), but effectivity can vary according to individual differences. Based on the available literature so far, electrode sizes do not appear to produce remarkable effectivity differences in this domain. However, since the antiproportional relationship between current density and electrode size (at fixed current strength) does not linearly scale for respective distances from the electrode (e.g., scalp-brain distance) and smaller electrodes may not reach deeper regions(Miranda et al. 2009), this observation will require systematic evaluation in the future. Furthermore, publication biases can impede the development of better stimulation models, because nonsignificant results are dismissed, theories are elaborated posthoc, and results could lack reproducibility. In this vein, preregistration of according studies and protocols (e.g., aspredicted.org or www.osf.io) could facilitate scientific communication and rigorous evaluation of tES methods. 


\section{Conclusion}

Both tDCS and tRNS can enhance arithmetic capabilities in adult populations and could be promising tools for deviant performance populations. The current literature is coined by heterogeneity already in standard technical parameters such as montage, but effectivity estimation requires better standardization. Studies with (sub)clinical populations and children are needed to examine the usefulness of stimulation for at-risk groups. However, having said that, future research must consider their potentially different neurofunctional signatures, potential additional side effects, and neuroethical questions (Cohen Kadosh et al. 2012; Davis 2014). Documentation of (individualized) task-specific brain activity will potentially allow for predictive adjustment of tES configurations and better evaluation thereof, i.e., using fMRI (Clemens et al. 2013), EEG (Grabner et al. 2015), or NIRS (Snowball et al. 2013). Further tES precision could be obtained from considering a neurodevelopmental perspective, concurrent (individualized) imaging, relevant domain-related states, and theoretical optimization of task-related brain-structure correspondence in experimental and training studies.

\section{Acknowledgements}

Christina Artemenko and Thomas Dresler were funded by the LEAD Graduate School \& Research Network [GSC1028], a project of the Excellence Initiative of the German federal and state governments.

\section{References}

Antal A, Herrmann CS (2016) Transcranial Alternating Current and Random Noise Stimulation: Possible Mechanisms. Neural Plast. doi: 10.1155/2016/3616807 Arsalidou M, Taylor MJ (2011) Is 2+2=4? Meta-analyses of brain areas needed for numbers and calculations. Neuroimage 54:2382-93. doi:

10.1016/j.neuroimage.2010.10.009

Artemenko C, Moeller K, Huber S, Klein E (2015) Differential influences of unilateral tDCS over the intraparietal cortex on numerical cognition. Front Hum Neurosci 9:110. doi: 10.3389/fnhum.2015.00110 
Batsikadze G, Moliadze V, Paulus W, et al (2013) Partially non-linear stimulation intensity-dependent effects of direct current stimulation on motor cortex excitability in humans. J Physiol 591:1987-2000. doi:

10.1113/jphysiol.2012.249730

Battleday RM, Muller T, Clayton MS, Kadosh RC (2014) Mapping the mechanisms of transcranial alternating current stimulation: A pathway from network effects to cognition. Front Psychiatry 5:162. doi: 10.3389/fpsyt.2014.00162

Bikson M, Datta A, Rahman A, Scaturro J (2010) Electrode montages for tDCS and weak transcranial electrical stimulation: Role of "return" electrode's position and size. Clin Neurophysiol 121:1-1976-1978. doi:

10.1097/MPG.0b013e3181a15ae8.Screening

Bikson M, Rahman A (2013) Origins of specificity during tDCS: anatomical, activityselective, and input-bias mechanisms. Front Hum Neurosci 7:688. doi: 10.3389/fnhum.2013.00688

Bynner J, Parsons S (1997) Does Numeracy Matter? Evidence from the National Child Development Study on the Impact of Poor Numeracy on Adult Life. Basic Skills Agency, Commonwealth House, London WC1A 1NU, London, United Kingdom

Cappelletti M, Gessaroli E, Hithersay R, et al (2013) Transfer of Cognitive Training across Magnitude Dimensions Achieved with Concurrent Brain Stimulation of the Parietal Lobe. J Neurosci 33:14899-14907. doi: 10.1523/JNEUROSCI.169213.2013

Chaieb L, Antal A, Paulus W (2015) Transcranial random noise stimulation-induced plasticity is NMDA-receptor independent but sodium-channel blocker and benzodiazepines sensitive. Front Neurosci 9:125. doi: 10.3389/fnins.2015.00125 Clemens B, Jung S, Zvyagintsev M, et al (2013) Modulating arithmetic fact retrieval: A 
single-blind, sham-controlled tDCS study with repeated fMRI measurements. Neuropsychologia 51:1279-1286. doi: 10.1016/j.neuropsychologia.2013.03.023 Cohen J (1988) Statistical Power analysis for the Behavioral Sciences, 2nd ed. Lawrence Erlbaum Associates, Inc., New Jersey

Cohen Kadosh R, Levy N, O'Shea J, et al (2012) The neuroethics of non-invasive brain stimulation. Curr Biol 22:R108-11. doi: 10.1016/j.cub.2012.01.013

Cohen Kadosh R, Soskic S, luculano T, et al (2010) Modulating neuronal activity produces specific and long-lasting changes in numerical competence. Curr Biol 20:2016-2020. doi: 10.1016/j.cub.2010.10.007

Davis NJ (2014) Transcranial stimulation of the developing brain: a plea for extreme caution. Front Hum Neurosci 8:8-11. doi: 10.3389/fnhum.2014.00600

Dehaene S, Piazza M, Pinel P, Cohen L (2003) Three parietal circuits for number processing. Cogn Neuropsychol 20:487-506. doi: 10.1080/02643290244000239

Dockery CA, Hueckel-Weng R, Birbaumer N, Plewnia C (2009) Enhancement of planning ability by transcranial direct current stimulation. J Neurosci 29:7271-7. doi: 10.1523/JNEUROSCI.0065-09.2009

Duecker F, Sack AT (2015) Rethinking the role of sham TMS. Front Psychol 6:1-5. doi: 10.3389/fpsyg.2015.00210

Fertonani A, Miniussi C (2016) Transcranial Electrical Stimulation: What We Know and Do Not Know About Mechanisms. Neurosci. doi: $10.1177 / 1073858416631966$

Fertonani A, Pirulli C, Miniussi C (2011) Random noise stimulation improves neuroplasticity in perceptual learning. J Neurosci 31:15416-23. doi: 10.1523/JNEUROSCI.2002-11.2011

Grabner RH, Rütsche B, Ruff CC, Hauser TU (2015) Transcranial direct current stimulation of the posterior parietal cortex modulates arithmetic learning. Eur $\mathrm{J}$ 
Neurosci 42:1667-1674. doi: 10.1111/ejn.12947

Hauser TU, Rotzer S, Grabner RH, et al (2013) Enhancing performance in numerical magnitude processing and mental arithmetic using transcranial Direct Current Stimulation (tDCS). Front Hum Neurosci 7:244. doi: 10.3389/fnhum.2013.00244 Hauser TU, Rütsche B, Wurmitzer K, et al (2016) Neurocognitive Effects of Transcranial Direct Current Stimulation in Arithmetic Learning and Performance: a Simultaneous tDCS-fMRI Study. Brain Stimul. doi: 10.1016/j.brs.2016.07.007 Houser R, Thoma S, Fonseca D, et al (2015) Enhancing statistical calculation with transcranial direct current stimulation (tDCS) to the left intra-parietal sulcus (IPS). Trends Neurosci Educ 4:98-101. doi: 10.1016/j.tine.2015.07.002 Houser R, Thoma S, Stanton M (2014) The effect of transcranial direct current stimulation (tDCS) on learning and performing statistical calculations. J Artic Support Null Hypothesis 11:1-10.

Iuculano T, Cohen Kadosh R (2013) The Mental Cost of Cognitive Enhancement. J Neurosci 33:4482-4486. doi: 10.1523/JNEUROSCI.4927-12.2013 Iuculano T, Cohen Kadosh R (2014) Preliminary evidence for performance enhancement following parietal lobe stimulation in Developmental Dyscalculia. Front Hum Neurosci 8:38. doi: 10.3389/fnhum.2014.00038 Jacobson L, Koslowsky M, Lavidor M (2012) tDCS polarity effects in motor and cognitive domains: a meta-analytical review. Exp brain Res 216:1-10. doi: $10.1007 / \mathrm{s} 00221-011-2891-9$

Kaufmann L, Mazzocco MM, Dowker A, et al (2013) Dyscalculia from a developmental and differential perspective. Front Psychol 4:1-5. doi: 10.3389/fpsyg.2013.00516

Klein E, Mann A, Huber S, et al (2013) Bilateral Bi-Cephalic tDCS with Two Active Electrodes of the Same Polarity Modulates Bilateral Cognitive Processes 
Differentially [corrected]. PLoS One 8:e71607. doi:

10.1371/journal.pone.0071607

Klein E, Suchan J, Moeller K, et al (2014) Considering structural connectivity in the triple code model of numerical cognition: differential connectivity for magnitude processing and arithmetic facts. Brain Struct Funct 1-17. doi: 10.1007/s00429014-0951-1

Krause B, Cohen Kadosh R (2013) Can transcranial electrical stimulation improve learning difficulties in atypical brain development? A future possibility for cognitive training. Dev Cogn Neurosci 6:176-194. doi:

10.1016/j.dcn.2013.04.001

Li LM, Leech R, Scott G, et al (2015) The effect of oppositional parietal transcranial direct current stimulation on lateralized brain functions. Eur J Neurosci 42:29042914. doi: 10.1111/ejn.13086

Looi CY, Cohen Kadosh R (2016) Brain stimulation , mathematical and numerical training : Contribution of core and non-core skills. In: Progress in brain research. pp 353-88

Looi CY, Duta M, Brem A-K, et al (2016a) Combining brain stimulation and video game to promote long-term transfer of learning and cognitive enhancement. Sci Rep 6:22003. doi: 10.1038/srep22003

Looi CY, Thompson J, Krause B, Cohen Kadosh R (2016b) The Neuroscience of Mathematical Cognition and Learning. Paris

Matejko AA, Ansari D (2015) Drawing connections between white matter and numerical and mathematical cognition: A literature review. Neurosci Biobehav Rev 48:35-52. doi: 10.1016/j.neubiorev.2014.11.006

Miranda PC, Faria P, Hallett M (2009) What does the ratio of injected current to electrode area tell us about current density in the brain during tDCS? Clin 
Neurophysiol 120:1183-1187. doi: 10.1016/j.clinph.2009.03.023

Moliadze V, Antal A, Paulus W (2010) Electrode-distance dependent after-effects of transcranial direct and random noise stimulation with extracephalic reference electrodes. Clin Neurophysiol 121:2165-71. doi: 10.1016/j.clinph.2010.04.033

Moliadze V, Fritzsche G, Antal A (2014) Comparing the efficacy of excitatory transcranial stimulation methods measuring motor evoked potentials. Neural Plast 2014:Article ID 837141. doi: 10.1155/2014/837141

Moyer RS, Landauer TK (1967) Time required for Judgements of Numerical Inequality. Nature 215:1519-1520. doi: 10.1038/2151519a0

Mulquiney PG, Hoy KE, Daskalakis ZJ, Fitzgerald PB (2011) Improving working memory: Exploring the effect of transcranial random noise stimulation and transcranial direct current stimulation on the dorsolateral prefrontal cortex. Clin Neurophysiol 122:2384-2389. doi: 10.1016/j.clinph.2011.05.009

Nasseri P, Nitsche MA, Ekhtiari H (2015) A framework for categorizing electrode montages in transcranial direct current stimulation. Front Hum Neurosci 9:1-5. doi: 10.3389/fnhum.2015.00054

Nieder A (2016) The neuronal code for number. Nat Rev Neurosci. doi: 10.1038/nrn.2016.40

Nitsche MA, Paulus W (2000) Excitability changes induced in the human motor cortex by weak transcranial direct current stimulation. J Physiol 527 Pt 3:633-9.

Ozen S, Sirota A, Belluscio MA, et al (2010) Transcranial Electric Stimulation Entrains Cortical Neuronal Populations in Rats. J Neurosci 30:11476-11485. doi:

10.1523/JNEUROSCI.5252-09.2010

Pasqualotto A (2016) Transcranial random noise stimulation benefits arithmetic skills. Neurobiol Learn Mem 133:7-12. doi: 10.1016/j.nlm.2016.05.004 Plewnia C, Schroeder PA, Kunze R, et al (2015) Keep Calm and Carry On: Improved 
Frustration Tolerance and Processing Speed by Transcranial Direct Current Stimulation (tDCS). PLoS One 10:e0122578. doi: 10.1371/journal.pone.0122578 Pope PA, Brenton JW, Miall RC (2015) Task-Specific Facilitation of Cognition by Anodal Transcranial Direct Current Stimulation of the Prefrontal Cortex. Cereb Cortex 1-8. doi: 10.1093/cercor/bhv094

Pope PA, Miall RC (2012) Task-specific facilitation of cognition by cathodal transcranial direct current stimulation of the cerebellum. Brain Stimul 5:84-94. doi: 10.1016/j.brs.2012.03.006

Popescu T, Krause B, Terhune DB, et al (2016) Transcranial random noise stimulation mitigates increased difficulty in an arithmetic learning task. Neuropsychologia 81:255-264. doi: 10.1016/j.neuropsychologia.2015.12.028

Priori A, Hallett M, Rothwell JC (2009) Repetitive transcranial magnetic stimulation or transcranial direct current stimulation? Brain Stimul 2:241-245. doi: 10.1016/j.brs.2009.02.004

Rahman A, Reato D, Arlotti M, et al (2013) Cellular effects of acute direct current stimulation: somatic and synaptic terminal effects. J Physiol 591:2563-78. doi: 10.1113/jphysiol.2012.247171

Rütsche B, Hauser TU, Jäncke L, Grabner RH (2015) When problem size matters: Differential effects of brain stimulation on arithmetic problem solving and neural oscillations. PLoS One 10:1-17. doi: 10.1371/journal.pone.0120665

Salillas E, Semenza C (2015) Mapping the Brain for Math: Reversible inactivation by direct cortical electrostimulation and transcranial magnetic stimulation. In: Cohen Kadosh R, Dowker A (eds) The Oxford Handbook of Numerical Cognition. Oxford University Press, New York, USA, pp 583-611

Sarkar A, Dowker A, Kadosh RC (2014) Cognitive Enhancement or Cognitive Cost: Trait-Specific Outcomes of Brain Stimulation in the Case of Mathematics Anxiety. 
J Neurosci 34:16605-16610. doi: 10.1523/JNEUROSCI.3129-14.2014

Schroeder PA, Pfister R, Kunde W, et al (2016) Counteracting Implicit conflicts by

Electrical Inhibition of the Prefrontal Cortex. J Cogn Neurosci 28:1737-1748. doi:

10.1162/jocn

Silvanto J, Pascual-Leone A (2008) State-dependency of transcranial magnetic stimulation. Brain Topogr 21:1-10. doi: 10.1007/s10548-008-0067-0

Snowball A, Tachtsidis I, Popescu T, et al (2013) Long-term enhancement of brain function and cognition using cognitive training and brain stimulation. Curr Biol 23:987-992. doi: 10.1016/j.cub.2013.04.045

Suárez-Pellicioni, Macarena, Núñez-Peña, María Isabel, \& Colomé À (2016) Math anxiety: A review of its cognitive consequences, psychophysiological correlates, and brain bases. Cogn Affect Behav Neurosci 16:3-22. doi: 10.3758/s13415015-0370-7

Terney D, Chaieb L, Moliadze V, et al (2008) Increasing human brain excitability by transcranial high-frequency random noise stimulation. J Neurosci 28:1414714155. doi: 10.1523/JNEUROSCI.4248-08.2008

Truong DQ, Hüber M, Xie X, et al (2014) Clinician accessible tools for GUI computational models of transcranial electrical stimulation: BONSAI and SPHERES. Brain Stimul 7:521-524. doi: 10.1016/j.brs.2014.03.009.Clinician Tzelgov J, Yehene V, Kotler L, Alon a (2000) Automatic comparisons of artificial digits never compared: learning linear ordering relations. J Exp Psychol Learn Mem Cogn 26:103-120. doi: 10.1037/0278-7393.26.1.103

van der Groen O, Wenderoth N (2016) Transcranial Random Noise Stimulation of Visual Cortex: Stochastic Resonance Enhances Central Mechanisms of Perception. J Neurosci 36:5289-98. doi: 10.1523/JNEUROSCI.4519-15.2016 Vanderhasselt M-A, De Raedt R, Namur V, et al (2015) Transcranial electric 
stimulation and neurocognitive training in clinically depressed patients: A pilot study of the effects on rumination. Prog Neuro-Psychopharmacology Biol Psychiatry 57:93-99. doi: 10.1016/j.pnpbp.2014.09.015

Zamarian L, Ischebeck A, Delazer M (2009) Neuroscience of learning arithmeticEvidence from brain imaging studies. Neurosci Biobehav Rev 33:909-925. doi: 10.1016/j.neubiorev.2009.03.005 
Table 1. Overview of original tES studies on numerosity and arithmetic performance, learning, and training. The majority of studies administered tES online to the behavioral task, in healthy student populations (mixed-sex aged 18-42 y) and deviations from these parameters are explicitly stated below. Anode and cathode target regions refer to the international 10-20 system for electrode placements. For tRNS, both anode and cathode deliver current (reported: peakto-peak intensity) in negative and positive direction and are assigned randomly (no study employed DC offset).

\begin{tabular}{|c|c|c|c|c|c|c|}
\hline Study & $\begin{array}{l}\text { tES Method } \\
\text { (group), anode, cathode, current } \\
\text { Duration, electrode size, } A C \text { range }\end{array}$ & Sample \& Design & Task & $\begin{array}{l}\text { Result } \\
+ \text { physiological effects } \\
\text { \# transfer, additional measures }\end{array}$ & Effect Size & Successful Montage \\
\hline \multicolumn{7}{|c|}{$\begin{array}{l}\text { 1. Cognitive Enhancement of Performance } \\
\text { 1.1. Numerosity }\end{array}$} \\
\hline Li et al., 2015 & $\begin{array}{l}\text { tDCS online } \\
\text { (LA-RC) P3, P4, 2mA } \\
\text { (RA-LC) P4, P3, 2mA } \\
\text { (S) sham } \\
30 \mathrm{~min}, 5 \times 5 \mathrm{~cm}\end{array}$ & $\begin{array}{l}\mathrm{N}=18 \\
\text { cross-over }\end{array}$ & $\begin{array}{l}\text { number comparison } \\
\text { spatial attention (modified } \\
\text { Posner task) } \\
\text { continuous attention, } \\
\text { vigilance level }\end{array}$ & $\begin{array}{l}(\mathrm{S})>(\mathrm{RA}-\mathrm{LC})[\mathrm{RT}] \\
\text { no modulation of spatial } \\
\text { attention } \\
(\mathrm{RC}-\mathrm{LA})>(\mathrm{RA}-\mathrm{LC})[\mathrm{RT} \text { in } \\
\text { final CRT block) }\end{array}$ & $d=0.61$ & \\
\hline $\begin{array}{l}\text { Schroeder, Pfister, Kunde, } \\
\text { Nuerk, \& Plewnia, } 2016\end{array}$ & $\begin{array}{l}\text { tDCS online } \\
\text { (1) extracephalic, F3, } 1 \mathrm{~mA} \text { and sham } \\
\text { (2) F3, extracephalic, } 1 \mathrm{~mA} \text { and sham } \\
25 \mathrm{~min}, 5 \times 7 \mathrm{~cm}\end{array}$ & $\begin{array}{l}N_{1}=24, N_{\text {rep }}=24, N_{2}=24 \\
\text { separate cross-over }\end{array}$ & $\begin{array}{l}\text { number-space } \\
\text { representation (SNARC) } \\
\text { stimulus-response conflict }\end{array}$ & $\begin{array}{l}\text { (1) reduces representation: } \\
\text { prefrontal areas direct } \\
\text { implicit number-space link } \\
\text { no effect on explicit conflict } \\
\text { no modulation of NDE [RTs] } \\
\text { \#conceptual replication }\end{array}$ & $d=0.49$ & \\
\hline \multicolumn{7}{|l|}{ 1.2. Arithmetic } \\
\hline $\begin{array}{l}\text { Artemenko, Moeller, Huber, \& } \\
\text { Klein, } 2015\end{array}$ & $\begin{array}{l}\text { tDCS online } \\
\text { (LA) P3, SO, } 1 \mathrm{~mA} \\
\text { (RA) P4, SO, 1mA } \\
\text { (LC) SO, P3,1mA } \\
\text { (RC) SO, P4, 1mA } \\
\text { (S) sham } \\
20 \text { min, } 5 \times 5 \text { parietal \& } 10 \times 10 \mathrm{~cm} \text { SO }\end{array}$ & $\begin{array}{l}\mathrm{N}=25 \\
\text { cross-over }\end{array}$ & $\begin{array}{l}\text { addition 2AFC } \\
\text { color-word stroop (offline } \\
\text { control) }\end{array}$ & $\begin{array}{l}\text { carry effect: RA > RC } \\
\text { no effect on stroop } \\
\text { place-value lateralized in } \\
\text { right IPS }\end{array}$ & $d=0.36$ & \\
\hline $\begin{array}{l}\text { Clemens, Jung, Zvyagintsev, } \\
\text { Domahs, \& Willmes, } 2013\end{array}$ & $\begin{array}{l}\text { tDCS online } \\
\text { (1) CP4, SO, } 2 \mathrm{~mA} \text { and sham } \\
20 \mathrm{~min}, 5 \times 7 \mathrm{~cm}\end{array}$ & $\begin{array}{l}\mathrm{N}=10 \text { male } \\
\text { within (pre-post) }\end{array}$ & $\begin{array}{l}\text { simple multiplication } \\
\text { verification }\end{array}$ & $\begin{array}{l}\text { no behavioral effect [RT/PE] } \\
\text { +pre- and post fMRI: } \\
\text { +AG activity increase }\end{array}$ & $d=1.85-2.941$ & \\
\hline Gill, Shah-Basak, Hamilton, 2015 & $\begin{array}{l}\text { tDCS offline } \\
\text { (1) } \mathrm{F} 3, \mathrm{SO}, 2 \mathrm{~mA} \text { and sham } \\
20 \mathrm{~min}, 5 \times 5 \mathrm{~cm}\end{array}$ & $\begin{array}{l}\mathrm{N}=22 \text { (collapsed) } \\
\text { cross-over }\end{array}$ & $\begin{array}{l}\text { PASAT addition } \\
\text { Preceding easy or difficult } \\
\text { verbal WM task with } \mathrm{tDCS}\end{array}$ & $\begin{array}{l}\text { better accuracy for fast } \\
\text { additions if preceded by } \\
\text { difficult WM task (3-Back) }\end{array}$ & $d=1.82$ & \\
\hline
\end{tabular}




\begin{tabular}{|c|c|c|c|c|c|c|}
\hline Hauser et al., 2016 & $\begin{array}{l}\text { tDCS online } \rightarrow \text { offline } \\
\text { (1) P5-CP5, SO, } 1 \mathrm{~mA} \text { or (2) sham } \\
\text { first } 30 \text { min of task, } 5 \times 7 \mathrm{~cm} \text { anode \& } \\
5 \times 10 \mathrm{~cm} \text { cathode }\end{array}$ & $\begin{array}{l}\mathrm{N}_{1}=20, \mathrm{~N}_{2}=20 \\
\text { between }\end{array}$ & $\begin{array}{l}\text { subtraction } 3 A F C \\
\text { repeated vs. novel problems }\end{array}$ & $\begin{array}{l}\text { no group differences in } \\
\text { learning and problem } \\
\text { solving (ceiling effect) } \\
\text { +tDCS nullified novelty- } \\
\text { +related right prefrontal } \\
\text { +deactivation [fMRI] }\end{array}$ & $d=2.01$ & and \\
\hline Houser, Thoma, \& Stanton, 2014 & $\begin{array}{l}\text { tDCS offline } \\
\text { (1) P3, F3, sham, (2) 1mA, or (3) } 2 \mathrm{~mA} \\
20 \mathrm{~min}, 5 \times 7 \mathrm{~cm}\end{array}$ & $\begin{array}{l}N_{1}=14, N_{2}=15, N_{3}=13 \\
\text { between }\end{array}$ & $\begin{array}{l}\text { statistical problem } \\
\text { (calculation of non- } \\
\text { parametric-Kruskal-Wallis) }\end{array}$ & $\begin{array}{l}\text { sham }=1 \mathrm{~mA}, 1 \mathrm{~mA}>2 \mathrm{~mA} \\
\text { Instruction and calculation } \\
\text { time covary with task } \\
\text { success; possible } 2 \mathrm{~mA} \\
\text { effect of prefrontal cathode }\end{array}$ & $d=0.4$ & \\
\hline $\begin{array}{l}\text { Houser, Thoma, Fonseca, } \\
\text { O'Connor, \& Stanton, } 2015\end{array}$ & $\begin{array}{l}\text { tDCS offline } \\
\text { (1) P3, T4, sham, (2) 1mA, or (3) } 2 \mathrm{~mA} \\
20 \mathrm{~min}, 3 \times 5 \mathrm{~cm}\end{array}$ & $\begin{array}{l}N_{1}=13, N_{2}=10, N_{3}=9 \\
\text { between }\end{array}$ & $\begin{array}{l}\text { statistical problem } \\
\text { (calculation of non- } \\
\text { parametric-Kruskal-Wallis) }\end{array}$ & $1 m A \& 2 m A>$ sham & $d=1.51-1.281$ & \\
\hline Klein et al., 2013 & $\begin{array}{l}\text { Bi-tDCS online } \\
\text { (1) } \mathrm{P} 3+\mathrm{P} 4, \mathrm{SO}+\mathrm{SO}, 1 \mathrm{~mA} \\
\text { (2) } \mathrm{SO}+\mathrm{SO}, \mathrm{P} 3+\mathrm{P} 4,1 \mathrm{~mA} \\
\text { (3) sham } \\
20 \mathrm{~min}, 5 \times 5 \text { parietal \& } 10 \times 10 \mathrm{~cm} \mathrm{SO}\end{array}$ & $\begin{array}{l}\mathrm{N} 24 \\
\text { cross-over }\end{array}$ & $\begin{array}{l}\text { addition 2AFC } \\
\text { color-word Stroop (control) }\end{array}$ & $\begin{array}{l}(1)<(2) \text { (distractor distance) } \\
\text { no effect on stroop } \\
\text { bilateral IPS for number } \\
\text { magnitude processing }\end{array}$ & $d=0.46^{1}$ & \\
\hline Pasqualotto, 2016 & $\begin{array}{l}\text { tRNS online } \\
\text { (F) F4, F3,1 } \mathrm{mA} \\
\text { (P) P4, P3,1 } \\
\text { (S) sham } \\
20 \mathrm{~min}, 5 \times 5 \mathrm{~cm}, A C: 100-600 \mathrm{~Hz}\end{array}$ & $\begin{array}{l}N_{F}=18, N_{P}=18, N_{S}=18 \\
\text { within-between }\end{array}$ & $\begin{array}{l}\text { subtraction verification } \\
\text { word categorisation }\end{array}$ & $\begin{array}{l}(\mathrm{F})=(\mathrm{P})>(\mathrm{S}) \text { Improvement } \\
{[\mathrm{RT} \text { block } 4-1]} \\
\text { 1w follow-up: }(\mathrm{F})=(\mathrm{P})>\mathrm{S}[\mathrm{PE} \\
\text { trained and novel problems] } \\
\text { no effect on categorisation }\end{array}$ & $\begin{array}{l}- \\
-\end{array}$ & \\
\hline Plewnia et al., 2015 & $\begin{array}{l}\text { tDCS online } \\
\text { (1) F3, extracephalic, } 1 \mathrm{~mA} \text { and sham } \\
20 \mathrm{~min}, 5 \times 7 \mathrm{~cm}\end{array}$ & $\begin{array}{l}\mathrm{N}=28 \text { male } \\
\text { between }\end{array}$ & PASAT addition & $\begin{array}{l}\text { Improved performance } \\
\text { Decreased negative affect }\end{array}$ & $\begin{array}{l}d=0.94 \\
d=1.37\end{array}$ & \\
\hline Pope et al., 2012 & $\begin{array}{l}\text { tDCS offline } \\
\text { (A) cerebellum, extracephalic, } 2 \mathrm{~mA} \\
\text { (C) extracephalic, cerebellum, } 2 \mathrm{~mA} \\
\text { (S) sham } \\
20 \mathrm{~min}, 5 \times 5 \mathrm{~cm}\end{array}$ & $\begin{array}{l}N_{A}=22, N_{c}=22, N_{s}=22 \\
\text { between (pre-post) }\end{array}$ & $\begin{array}{l}\text { PASAT addition and } \\
\text { subtraction; verb generation }\end{array}$ & $\begin{array}{l}(C)>(S)=(A) \text { for subtraction } \\
\text { and verb generation }\end{array}$ & - & \\
\hline
\end{tabular}


Pope et al., 2015

Rütsche, Hauser, Jäncke, \& Grabner, 2015
tDCS offline

(A) F3, extracephalic, $2 \mathrm{~mA}$

(S) sham

$20 \mathrm{~min}, 5 \times 5 \mathrm{~cm}$

\section{tDCS offline}

1) $\mathrm{P} 5-\mathrm{CP} 5, \mathrm{SO}, 1.5 \mathrm{~mA}$ and sham $30 \mathrm{~min}, 5 \times 7$ parietal \& $10 \times 10 \mathrm{~cm} \mathrm{SO}$

$\mathrm{N}=23$

cross-over
(C) extracephalic, F3, $2 \mathrm{~mA}$
$\mathrm{N}_{A}=20, \mathrm{~N}_{\mathrm{C}}=20, \mathrm{~N}_{\mathrm{S}}=19$

between (pre-post)

PASAT addition and

subtraction

small arithmetic problems

large arithmetic problems (verbal production)

\section{Nhigh math anxiety $=25$ \\ Nlow math anxiety $=20$}

mixed cross-over affective priming+ arithmetic

verification,

attention (ANT)
$(A)>(S)=(C)$ for subtraction

and verb generation

decreased performance PE

increased performance RT

differential involvement of

LPPC in arithmetic

problems; state dependency

+EEG during task:

+alpha \& theta modulations

RT benefit for high anxiety,

RT impaired for low anxiety

and executive control score

+modulation of salivary

+cortisol (pre-post)

\subsection{Numerosity \& Arithmetic}

Hauser, Rotzer, Grabner,

Mérillat, \& Jäncke, 2013: Exp. 1

(Bi-)tDCS offline

(1) $\mathrm{P} 3, \mathrm{SO}, 1 \mathrm{~mA}$

(2) $\mathrm{P} 3+\mathrm{P} 4, \mathrm{SO}, 1 \mathrm{~mA}$

(3) $\mathrm{SO}, \mathrm{P} 3+\mathrm{P} 4,1 \mathrm{~mA}$

(4) sham

$25 \mathrm{~min}, 5 \times 7$ target \& $10 \times 10 \mathrm{~cm}$ return

electrodes

Hauser, Rotzer, Grabner

tDCS offline

Mérillat \& Jäncke, 2013. Exp. 2

(1) $\mathrm{P} 4, \mathrm{SO}, 1 \mathrm{~mA}$ and $\mathrm{sham}$

$25 \mathrm{~min}, 5 \times 7$ target $\& 10 \times 10 \mathrm{~cm}$ retum electrodes

\section{Cognitive Enhancement of Training and Learning}

\subsection{Numerosity}

Cappelletti et al., 2013

$\begin{array}{ll}\text { tRNS online } \rightarrow \text { offline } & N_{P}=10, N_{P N T}=10 \\ \text { (P) P3, P4, 1mA } & N_{M}=10, N_{S T}=10 \\ \text { (PNT) P3, P4, 1mA, no training } & \text { between } \\ \text { (M) C3, C4, 1mA } & \end{array}$

(M) C3, C4, 1mA

(ST) sham \& training

20 min for $5 d, 5 \times 7 \mathrm{~cm}, A C: 0-250 \mathrm{~Hz}$
$\mathrm{N}=21$

cross-over

number comparison

subtraction $3 \mathrm{AFC}$

$\mathrm{N}=16$

cross-over

subtraction $3 \mathrm{AFC}$
P3 > sham $[P E]$

P3 > sham [RT]

unilateral left parietal tDCS

enhanced performance

no modulation of NDE

\section{$\mathrm{d}=0.51$}

$d=0.45$

$d=0.47-0.72$

$d=5.691$

$d=4.13^{1}$

$d=1.03^{1}$

no effects (control

experiment; laterality specificity)

number acuity: dot-array numerosity

discrimination training $(5 \mathrm{~d})$

transfer: continuous

arithmetic, attention,

executive, Stroop (control) magnitudes discrimination,

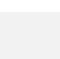

$\mathrm{P}>\mathrm{M}=\mathrm{ST}>\mathrm{PNT}$

$(\mathrm{P})$ improved continuous

magnitude discrimination

no effects on arithmetic \&

control tasks

$+(\mathrm{P})$ displayed sustained

+discrimination acuity after

+16 weeks $d=1.12-1.25^{1}$

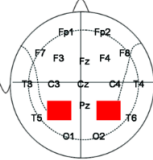

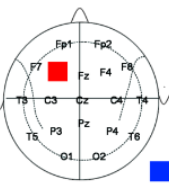




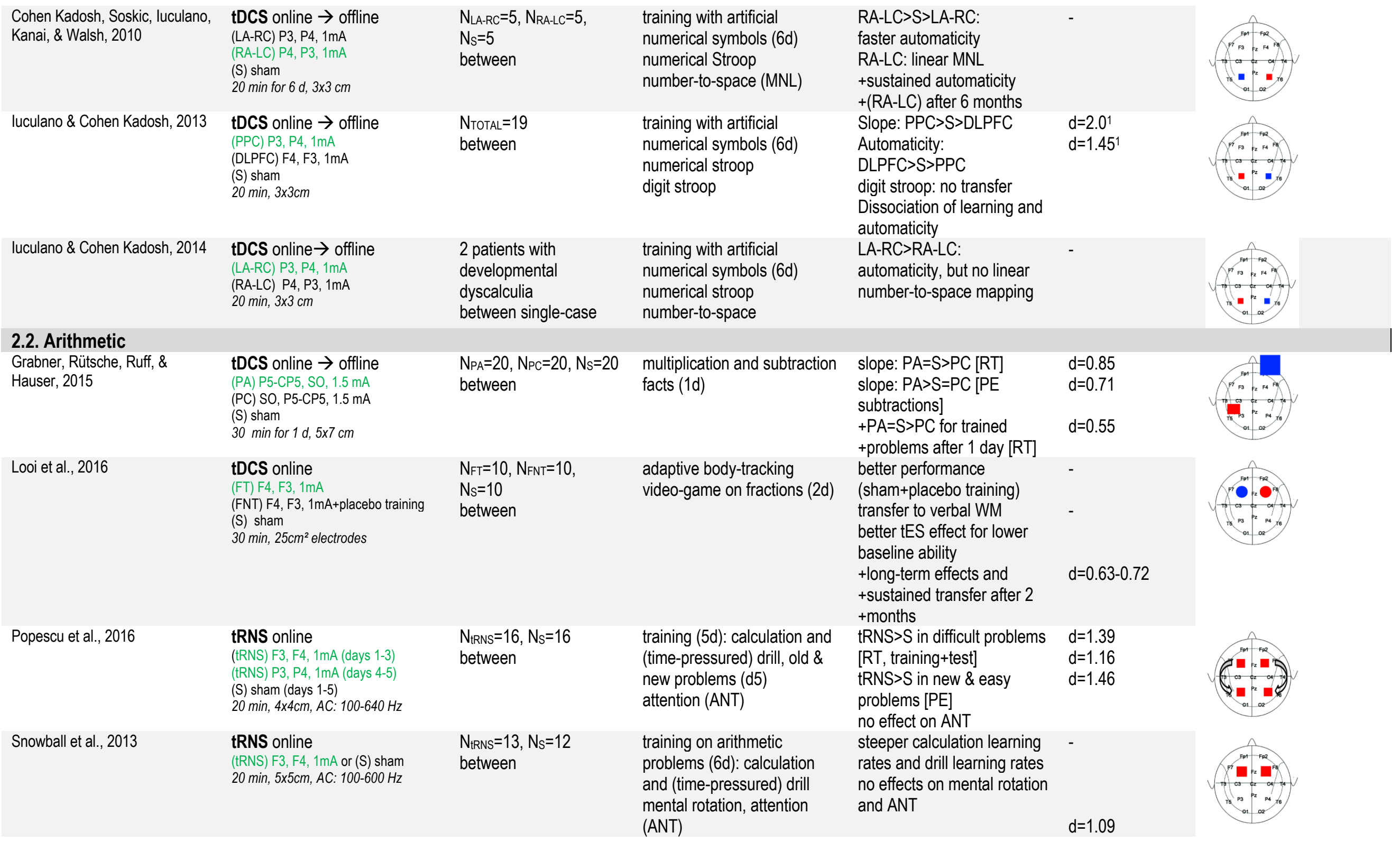




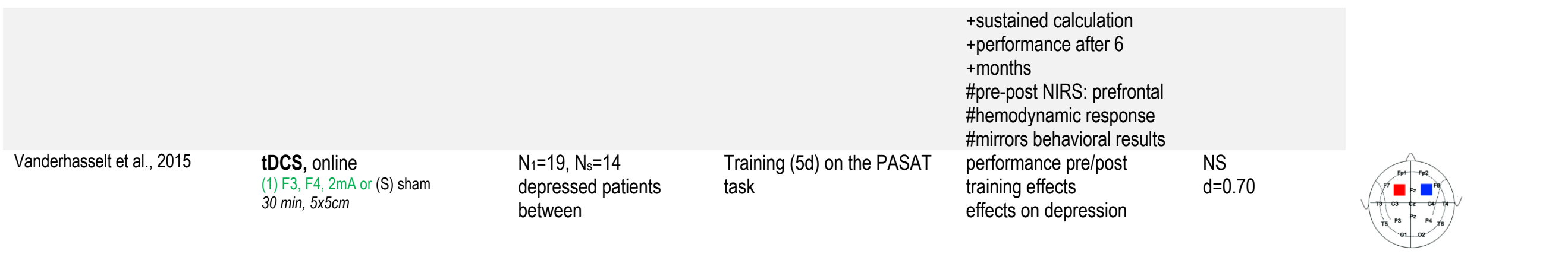

Notes: 2AFC=two alternatives forced choiceAC=alternating current, AG=angular gyrus, IPS=intraparietal sulcus, NDE=numerical distance effect, PASAT=Paced Auditory Serial Addition Task, PE=percentage of errors, $\mathrm{PFC}=$ prefrontal cortex, $\mathrm{RT}=$ response times, $\mathrm{SO}=$ (contralateral) supraorbital region. Cross-over design refers to repeated measurement of different stimulation conditions in counterbalanced order. Cohen's d: $0.2=$ small effect, $0.5=$ medium effect, $0.8=l a r g e$ effect. 1 Effect sizes are transformed following Cohen (1988) for two means and for more than two means with a significant linear trend. 